\title{
Enhanced thermoelectricity by controlled local structure in bismuth-chalcogenides
}

\author{
Cite as: J. Appl. Phys. 125, 145105 (2019); doi: 10.1063/1.5087096 \\ Submitted: 28 December 2018 - Accepted: 21 March 2019 . \\ Published Online: 10 April 2019
}

K. Terashima, ${ }^{1, a)}$ (D) Y. Yano, ${ }^{2, b)}$ E. Paris, ${ }^{3}$ (D) Y. Goto, ${ }^{4}$ (D) Y. Mizuguchi, ${ }^{4}$ Y. Kamihara, ${ }^{5}$ (D) T. Wakita, ${ }^{7}$ Y. Muraoka, ${ }^{1}$ N. L. Saini, ${ }^{3}$ iD and T. Yokoya

\author{
AFFILIATIONS \\ ${ }^{1}$ Research Institute for Interdisciplinary Science, Okayama University, Okayama 700-8530, Japan \\ ${ }^{2}$ Graduate School of Natural Sciences, Okayama University, Okayama 700-8530, Japan \\ ${ }^{3}$ Dipartimento di Fisica, Universitá di Roma "La Sapienza" - P. le Aldo Moro 2, 00185 Roma, Italy \\ ${ }^{4}$ Department of Physics, Tokyo Metropolitan University, Tokyo 192-0397, Japan \\ ${ }^{5}$ Department of Applied Physics and Physico-Informatics, Keio University, Yokohama 223-8522, Japan
}

\author{
a) Author to whom correspondence should be addressed: k-terashima@cc.okayama-u.ac.jp

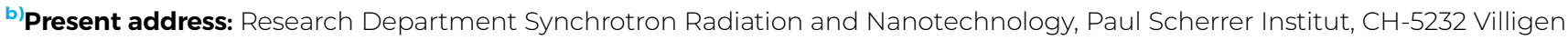 \\ PSI, Switzerland.
}

\begin{abstract}
Spectroscopic techniques, including photoelectron spectroscopy, diffuse reflectance, and x-ray absorption, are used to investigate the electronic structure and the local structure of $\mathrm{LaOBiS}_{2-x} \mathrm{Se}_{x}$ thermoelectric material. It is found that Se substitution effectively suppresses local distortion that can be responsible for the increased carrier mobility together with a change in the electronic structure. The results suggest a possible way to control thermoelectric properties by tuning of the local crystal structure of these materials.
\end{abstract}

Published under license by AIP Publishing. https://doi.org/10.1063/1.5087096

\section{INTRODUCTION}

Thermoelectric materials with layered structures hold possibilities to modify their physical properties, e.g., by partial substitutions in the block layer and/or the conducting layer. Among them, the $\mathrm{REOBiCh}_{2}(\mathrm{RE}=$ rare earth and $\mathrm{Ch}=$ chalcogen $)$ system $^{1}$ is acquiring much attention due to its structural similarity with high- $T_{c}$ cuprates and iron-based superconductors, in which the carrier number and other physical parameters are known to be controlled by combinations of constituting block layers and conducting layers. ${ }^{2-11}$ The parent material, $\mathrm{LaOBiCh}_{2}$, is insulating, and an object of superconductivity research since it becomes a superconductor by carrier doping. ${ }^{1}$ It was found ${ }^{12}$ that isovalent substitution of chalcogen atoms in the parent compound induces a large improvement of the thermoelectric figure of merit given by $Z T=\left(S^{2} / \rho \kappa\right) T$. The $Z T$ in the $\mathrm{LaOBiS}_{2-x} \mathrm{Se}_{x}$ system has been found ${ }^{14}$ to be as high for $\sim 0.36$ in $x=1.0$ at $650 \mathrm{~K}$.

The thermoelectric $Z T$ consists of several physical parameters, namely, Seebeck coefficient $(S)$, resistivity $(\rho)$, and thermal conductivity $(\kappa)$. For $x=1.0$, the absolute value of the Seebeck coefficient is $\sim 20 \%$ higher, while the total thermal conductivity remains similar to that of $x=0.0$; instead the resistivity shows a significant decrease. $^{12}$ It can be seen in Fig. 1 that the electrical resistivity of LaOBiSSe (corresponds to $x=1.0$ ) below room temperature is smaller than that of $\mathrm{LaOBiS}_{2}$ by about a factor of 10 . Judging from the reported relationship between the carrier concentration and the Seebeck coefficient in $\mathrm{LaOBiCh}_{2},{ }^{13}$ the carrier concentrations in $\mathrm{LaOBiS}_{2}$ and LaOBiSSe are expected to be similar. ${ }^{14}$ In a previous study, ${ }^{14}$ it has been argued that such a difference in electrical resistivity could be related with a large enhancement of carrier mobility by Se substitution, attributed to the chemical pressure effect, or more precisely, to the misfit strain effect in the layered structure, since the ionic radius of Se is larger than that of S. This may cause an enhanced overlap between adjacent orbitals through substitution, resulting in a change in the electronic structure. However, there is no direct experimental evidence providing the microscopic mechanism responsible for improving the thermoelectric properties in this system. This is also important due to the fact that $\mathrm{REOBiS}_{2}$ is structurally instable ${ }^{15}$ with polymorphism ${ }^{16,17}$ characterized by coexisting 


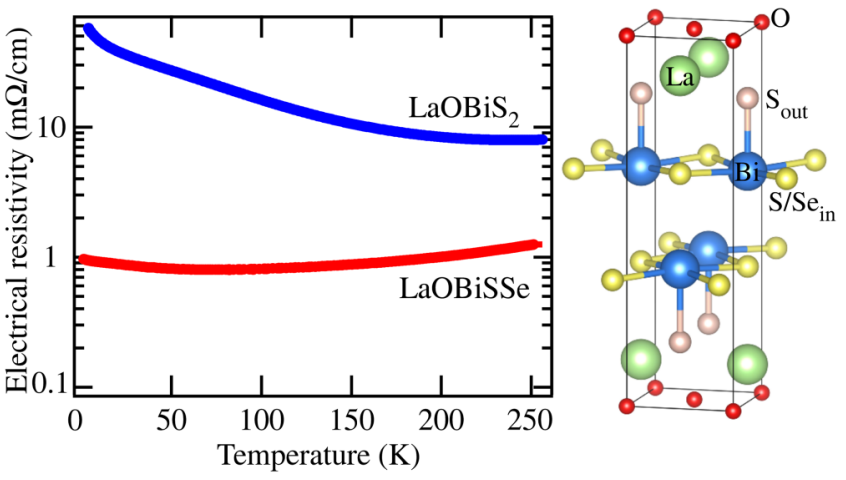

FIG. 1. Left: Temperature dependence of electrical resistivity of $\mathrm{LaOBiS}_{2}$ (blue) and LaOBiSSe (red). Right: Crystal structure of $\mathrm{LaOBiCh}_{2}$.

atomic configurations, and hence, apart from the knowledge of the electronic transport, the study of the local structure should provide an important feedthrough.

Here, we have performed a comparative study of the electronic and local structures of $\mathrm{LaOBiS}_{2}$ and $\mathrm{LaOBiSSe}$ using photoemission, diffuse reflectance, band calculation, and x-ray absorption fine structure, to address the possible origin of the enhanced carrier mobility by Se substitution. We have observed that the valence band structure is altered by Se substitution, with the bandgap decreasing by about half, which agrees qualitatively with first-principle band structure calculations. According to band structure calculations, the top of the valence band mainly consists of in-plane chalcogen $p$-orbitals which are strongly affected by partial substitution of chalcogen atoms. The calculated conduction band does not show any substantial change with Se substitution, indicating that the large enhancement of carrier mobility below room temperature may not be accounted for the change in the electronic structure. On the other hand, the x-ray absorption fine structure shows a marked change by substitution, indicating that the local distortion of the in-plane Bi-Chalcogen plane in the LaOBiSSe compound is drastically suppressed with respect to $\mathrm{LaOBiS}_{2}$. It has been suggested that this suppression of the local distortion might contribute greatly to the enhanced thermoelectric property of this system.

\section{METHODS}

Polycrystalline $\mathrm{LaOBiS}_{2}$ and LaOBiSSe samples were prepared by the solid-state-reaction method. ${ }^{1,12}$ The same procedure was used for preparing the two samples with the same person as the former reports of the crystal structure and thermoelectric properties. ${ }^{14,18}$ Temperature dependence of electrical resistivity was measured by the four-probe method.

Photoelectron spectroscopy measurements on pelletized samples were performed using the Scienta-Omicron R4000 analyzer constructed in Okayama University. Xe $\mathrm{I} \alpha(h v=8.437 \mathrm{eV})$, He I $\alpha$ $(21.218 \mathrm{eV})$, and $\mathrm{He} \mathrm{II} \alpha(40.814 \mathrm{eV})$ lines were used to excite photoelectrons. The energy resolution was set to $10 \mathrm{meV}$ for Xe I and He I and $50 \mathrm{meV}$ for He II, and the spectra were taken by the transmission mode in an ultrahigh vacuum of $8 \times 10^{-9} \mathrm{~Pa}$. The Fermi energy $\left(E_{F}\right)$ of samples was calibrated by that of gold, electronically contacted with samples. During the measurements, we found no signature of charging. Clean surfaces for the measurements were obtained by in situ scraping of samples at $2 \times 10^{-8} \mathrm{~Pa}$.

Total diffuse reflectance spectra $(\mathrm{R})$ were measured by a spectrometer with an integrating sphere (Hitachi High-Tech, U-4100). $\mathrm{Al}_{2} \mathrm{O}_{3}$ powders were used as the standard reference. Optical absorption coefficient $(\alpha)$ was determined from the $R$ value via the Kubelka-Munk equation, $(1-R)^{2} / 2 R=\alpha / s$, where $s$ is the scattering factor.

Bi L $\mathrm{L}_{3}$-edge $(E=13418 \mathrm{eV}) \mathrm{x}$-ray absorption measurements were used to probe the local structure of $\mathrm{LaOBiS}_{2}$ and LaOBiSSe. The measurements were performed at the beamline BM26A of the European Synchrotron Radiation Facility (ESRF), where the synchrotron light was monochromatized using a double crystal $\mathrm{Si}(111)$ monochromator. The measurements were made sequentially at low temperature $(T=30 \mathrm{~K})$ in the transmission mode. The finely powdered samples of $\mathrm{LaOBiS}_{2}$ and LaOBiSSe were diluted uniformly in the boron nitride matrix and pressed into pellets of $13 \mathrm{~mm}$ diameter, for obtaining the edge jump to be about 1 . The EXAFS oscillations were extracted by the standard procedure based on the cubic spline fit to the pre-edge subtracted absorption spectrum. ${ }^{27}$

First-principles calculations were performed using the WIEN2k code, ${ }^{19}$ where spin-orbit coupling was included. The lattice parameters were taken from x-ray diffraction measurements on $\mathrm{LaOBiS}_{2}$ and LaOBiSSe reported earlier. ${ }^{18}$ The modified Becke-Johnson $(\mathrm{mBJ})$ potential proposed by Tran and Blaha ${ }^{20,21}$ was used as the exchange correlation functional, as it has been discussed to be appropriate for the $\mathrm{BiS}_{2}$ system. ${ }^{22}$ For simplicity, we assumed the tetragonal crystal structure ${ }^{23}$ despite a symmetry reduction in both compounds. ${ }^{24-26}$ A $24 \times 24 \times 7 k$-mesh was used, with the $R K_{\max }$ parameter being 7 .

\section{RESULTS AND DISCUSSION}

Firstly, we focus on the effect of Se substitution on the electronic structure. Figure 2 shows photoemission spectra of $\mathrm{LaOBiS}_{2}$ and LaOBiSSe measured using several ultraviolet photon energies at $T=300 \mathrm{~K}$. Spectra in Figs. 2(a) and 2(b) are normalized by total intensity over the shown area, while those in Fig. 2(c) are normalized by the peak height. The overall width of the valence band and the energy position of the top of the valence band in $\mathrm{LaOBiS}_{2}$ is consistent with our earlier soft $\mathrm{x}$-ray photoemission work. ${ }^{28}$ Through S substitution with Se, we have observed that the top of the valence band becomes closer to $E_{F}$ at all the photon energies. The inset of Fig. 2(c) shows the near- $E_{\mathrm{F}}$ spectra divided by the Fermi-Dirac function of $T=300 \mathrm{~K}$ convoluted by experimental energy resolution. We have found that the $E_{\mathrm{F}} \mathrm{s}$ of both samples are located at the bottom of the conduction band that would be consistent with the sign of the Seebeck coefficient. Therefore, the magnitude of the bandgap can be evaluated for both samples, which is roughly 1.1 and $0.6 \pm 0.2 \mathrm{eV}$ for $\mathrm{LaOBiS}_{2}$ and $\mathrm{LaOBiSSe}$, respectively. The reduction of the bandgap due to Se substitution is also verified by $(\alpha h v / s)^{2}$ measurements ${ }^{29}$ on $\mathrm{LaOBiS}_{2}$ and $\mathrm{LaOBiSSe}$ shown in Figs. 2(d) and 2(e), where direct-transition-type optical 
(a)

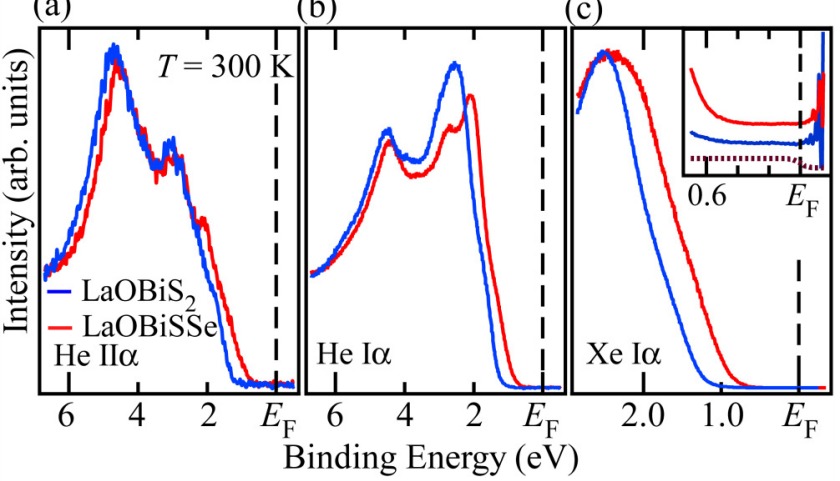

(d)

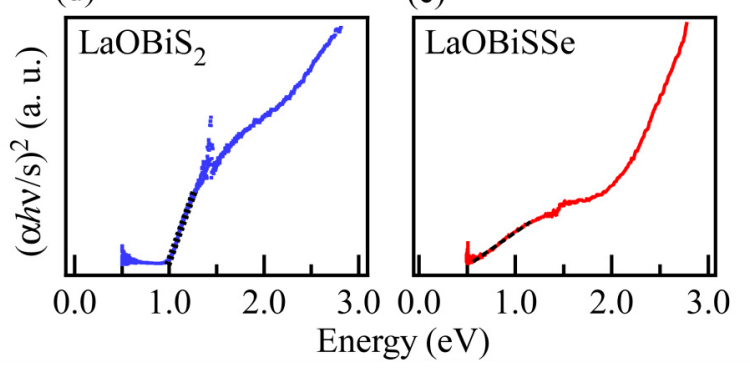

FIG. 2. Photoemission spectra of $\mathrm{LaOBiS}_{2}$ (blue) and LaOBiSSe (red) taken by (a) $\mathrm{He} \| \alpha$, (b) $\mathrm{He} \mathrm{l} \alpha$, and (c) $\mathrm{Xel} \alpha$ lines at $T=300 \mathrm{~K}$. The inset in (c) shows the spectra near $E_{\mathrm{F}}$ divided by the Fermi-Dirac function convoluted by experimental energy resolution (shown as dotted line). (d) and (e) $(\alpha h v / s)^{2}$ as a function of $h v$ on LaOBiS 2 and LaOBiSSe, respectively. The dotted lines in (d) and (e) are a guide for the eye.

absorption was observed for $1.0 \pm 0.1 \mathrm{eV}$ for $\mathrm{LaOBiS}_{2}$ and $0.5 \pm 0.1 \mathrm{eV}$ for LaOBiSSe.

In order to discuss the origin of the observed change in the electronic structure, we have performed band structure calculations including spin-orbit interaction. Upper panels of Figs. 3(a) and $3(\mathrm{~b})$ show calculated partial density of states (pDOSs) for $\mathrm{LaOBiS}_{2}$ (a) and LaOBiSSe (b), where $S_{\text {in }}$ denotes the in-plane $\mathrm{S}$ atom and $S_{\text {out }}$ is the out-of-plane $S$ atom. Following earlier experimental and theoretical works, ${ }^{18,30}$ we have assumed that Se atoms occupy only in-plane S sites. Bottom of Figs. 3(a) and 3(b) are experimental spectra (red lines), overlapped with simulated photoemission spectra (blue dashed lines), where each pDOS was multiplied by photoionization cross section ${ }^{31,32}$ and extended 1.3 times in energy for comparison. For calculated results, the position of $E_{F}$ was set at the bottom of the conduction band, in order to see the correspondence with the photoemission results in Fig. 2. The simulated spectra successfully captures the characteristics of Chalcogen-substitution induced change in the valence band. Namely, (i) the peak structure $\sim 2.5 \mathrm{eV}$ in $\mathrm{LaOBiS}_{2}$ splits into $\sim 2.5 \mathrm{eV}$ and $\sim 2 \mathrm{eV}$ in LaOBiSSe (ii) the top of the valence band becomes closer to $E_{F}$ and the bandgap decreases by Se substitution. Thus, it can be said that the first-principles calculations would give reliable prediction in these materials. (a)

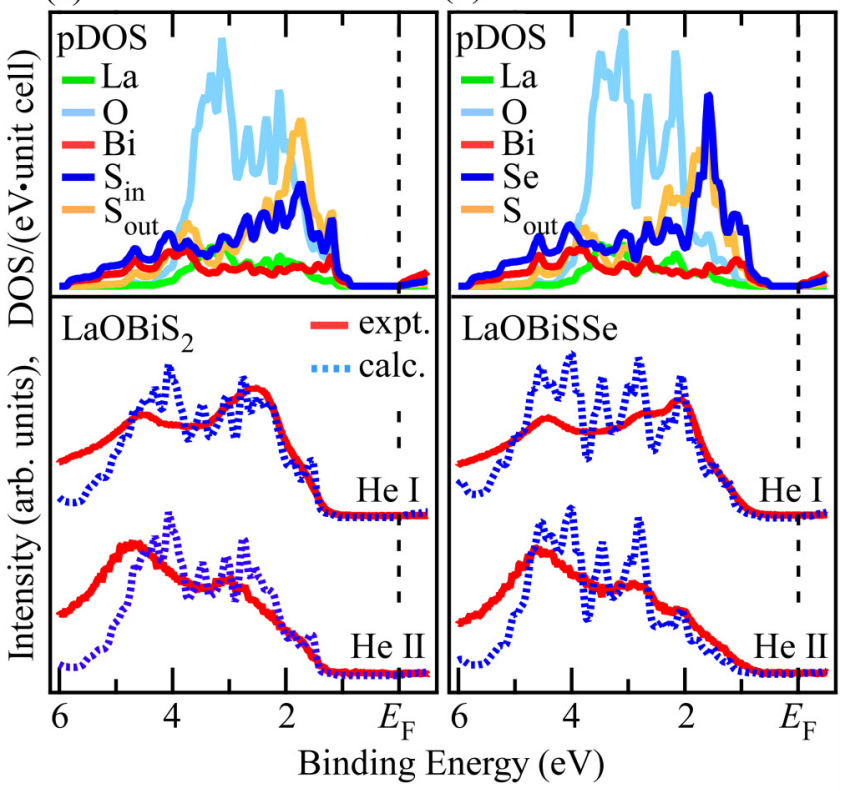

(c)

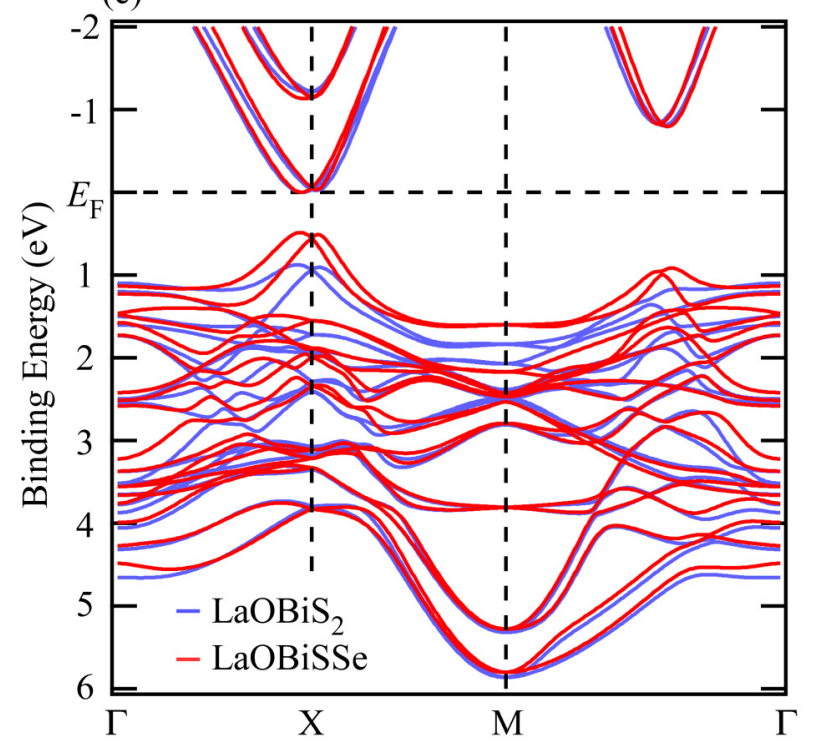

FIG. 3. (a) and (b) Top: Calculated pDOS for $\mathrm{LaOBiS}_{2}$ and LaOBiSSe. Bottom: Photoemission data (red lines) and simulated spectra (blue dashed lines) based on calculated pDOS and photoionization cross section. (c) Calculated band structure along high symmetry lines for $\mathrm{LaOBiS}_{2}$ (blue) and LaOBiSSe (red).

According to the calculations shown in Figs. 3(a) and 3(b), the changes in the experimental spectra are mainly attributed to the pDOS of the in-plane chalcogen atom. As shown in Fig. 3(c), it is expected that the top of the valence band is largely affected by the substitution of $\mathrm{S}$ with Se, while the bottom of the conduction band is less affected although the dispersion becomes slightly 
steeper in LaOBiSSe [estimated effective masses $\left(m^{*}\right)$ for the bottom of the conduction bands up to $0.1 \mathrm{eV}$ are 0.16 and 0.11 for $\mathrm{LaOBiS}_{2}$ and LaOBiSSe, respectively]. Such a change in the band structure would contribute the thermoelectric property especially at high temperature, whereas it would not fully explain the reduction of the resistivity in Fig. 1 since the chemical potential seems to be pinned at the bottom of the conduction band and the population of the Fermi-Dirac function at a binding energy of $0.5-1.0 \mathrm{eV}$ hardly changes below room temperature [see the inset of Fig. 2(c)]. Therefore, chalcogen substitution certainly modifies the band structure but the change would not fully account for the improvement of the thermoelectric property. Next, we focus on the Se-substitution effect on the local structure.

Figure 4 shows Fourier transforms (FTs) of $k^{2}$-weighted EXAFS oscillations extracted from the $\mathrm{Bi}_{3}$-edge $\mathrm{x}$-ray absorption spectra measured on $\mathrm{LaOBiS}_{2}$ and $\mathrm{LaOBiSSe}$ samples at $T=30 \mathrm{~K}$. The inset includes EXAFS oscillations ( $k^{2}$-weighted) as a function of photoelectron wave vector $k$. The FTs are performed using a gaussian window with the $k$ range being 3.5-15 $\AA^{-1}$ and corrected for the phase shifts. The EXAFS oscillations and the FT peak structures are largely different, revealing distinct local structure of the two compounds.

In the $\mathrm{LaOBiS}_{2}$ structure, the $\mathrm{Bi}$ atom is coordinated with one sulfur atom in the axial direction $\left(\mathrm{S}_{\text {out }}\right)$, four in-plane sulfur atoms $\left(S_{i n}\right)$, and the $S_{i n}$ atom of the adjacent $\mathrm{BiS}_{2}$ layer. Information on all these $\mathrm{Bi}-\mathrm{S}$ distances is contained in the main peak structure between 1.5 and $3.5 \AA$ in the FTs of $\mathrm{Bi}_{3}$-edge EXAFS. The contributions of distant atoms are mixed with multiple scatterings and visible beyond the first peak structure. To determine the local structure parameters, the EXAFS oscillations were modeled by

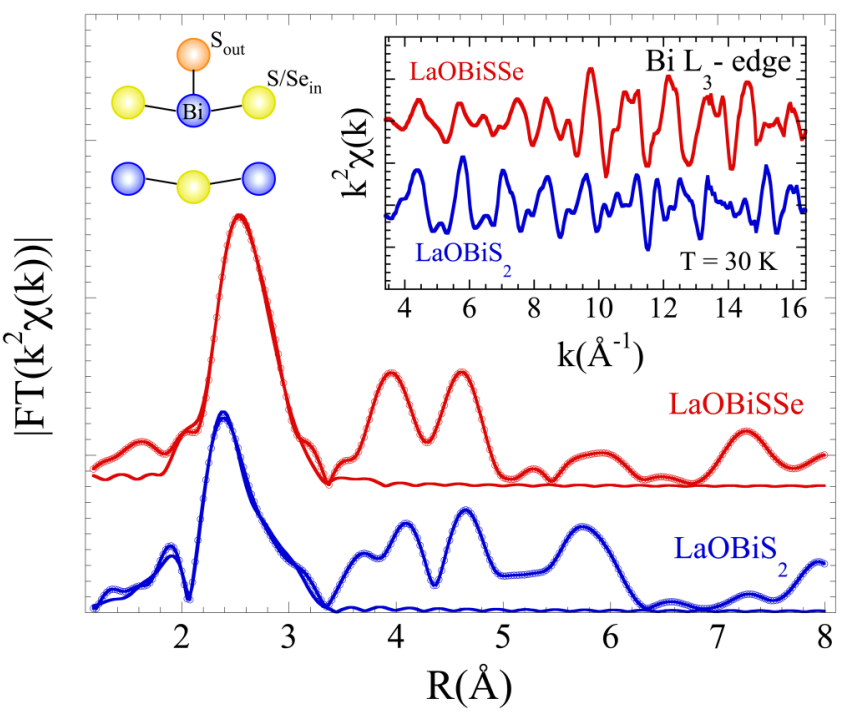

FIG. 4. Fourier transform (FT) magnitudes of the $\mathrm{Bi} L_{3}$-edge EXAFS of $\mathrm{LaOBiS}_{2}$ and LaOBiSSe. Model fits to the FTs are also shown as solid lines. EXAFS oscillations of the two samples and schematic view of atoms surrounding $\mathrm{Bi}$ are displayed as the inset. the general equation ${ }^{27}$ based on single-scattering approximation. Following earlier EXAFS study and theoretical calculations, ${ }^{16,33,34}$ we used two different in-plane Bi- $S_{i n}$ distances for $\mathrm{LaOBiS}_{2}$. The model contains four shells including one out-of-plane $\mathrm{Ch}_{\text {out }}$ atom (Bi-Chout distance), four in-plane $\mathrm{Ch}_{\text {in }}$ atoms at two different distances (two $\mathrm{Bi}-\mathrm{Ch}_{\text {in }}$ distances), and one $\mathrm{Bi}^{-} \mathrm{Ch}_{\text {in }}$ distance between the two $\mathrm{BiCh}_{2}$-layers. For $\mathrm{LaOBiSSe}$, considering the fact that $\mathrm{Se}$ preferentially occupies the $S_{\text {in }}$ site, ${ }^{18}$ Se occupation at the $S_{\text {out }}$ site was neglected. We have fixed the passive electrons reduction factor, $S_{0}^{2}=0.95$ for the analysis of the $\mathrm{Bi}_{3}$-edge EXAFS, while the photoelectron energy zero, $E_{0}$ was set to zero after analyzing different scans. The only parameters refined here are the local bondlengths and the associated $\sigma_{i}^{2}$, measuring the mean square relative displacement (MSRD) of the considered bond distances. The FEFF8 code ${ }^{35}$ was used for the calculation of scattering amplitudes and the WINXAS package ${ }^{36}$ was used for the EXAFS model fits. The fit $k$-range was $3.5-15 \AA^{-1}$, while $R$-range was $1.5-3.5 \AA$. Therefore, the number of independent points $2 \Delta k \Delta R / \pi$ was $\sim 14$ for the model fits in which the fit parameters were 8 to 10 . The model fits are included in Fig. 4 as solid lines.

The local structure parameters obtained for two compounds are summarized in Table I. The results on $\mathrm{LaOBiS}_{2}$ are consistent with earlier EXAFS work on the local structure of the F-doped system $^{33}$ and PDF analysis ${ }^{24}$ showing $\mathrm{Bi}-\mathrm{S}_{\text {in }}$ bondlength splitting. This confirms that the $\mathrm{BiS}_{2}$ layer is largely distorted in $\mathrm{LaOBiS}_{2}$. On the other hand, LaOBiSSe contains two $\mathrm{Bi}^{-} \mathrm{Se}_{\text {in }}$ distances separated by $\sim 0.2 \AA$. From Table I, it is apparent that local distortion in LaOBiSSe is substantially lower with respect to that in $\mathrm{LaOBiS}_{2}$. Therefore, it is likely that structural instability in $\mathrm{LaOBiS}_{2}$, characterized by polytypism, is partially suppressed in LaOBiSSe. Apart from other factors like microstructure, we think this could be the main origin of the change in the resistivity and hence the thermoelectric properties through chalcogen substitution, especially at lower temperature range.

The present EXAFS and earlier experimental works $s^{24,25,33}$ indicate that $\mathrm{Bi}$ atoms in $\mathrm{LaOBiS}_{2}$ are in the off-center position in $S_{\text {in }}$ square lattice. Such a lowering of the structural symmetry has been intensively studied in post-transition metal oxides and chalcogenides, ${ }^{37}$ where it turned out that the lone pair becomes active when the energy level of chalcogen $p$-orbitals are close enough to that of the $s$-orbital in the post-transition metal. If the hybridization between those two are strong enough, the post-transition

TABLE I. Bond distances $(\mathrm{R})$ and mean square relative displacements $\left(\sigma^{2}\right)$ of $\mathrm{LaOBiS}_{2}$ and LaOBiSSe determined by Bi $\mathrm{L}_{3}$-edge EXAFS $(T=30 \mathrm{~K})$. Maximum uncertainty in distance determination is about $\sim 0.01 \AA$, and in the corresponding $\sigma^{2}$ is about $\sim 0.001 \AA^{2} . \mathrm{Bi}^{-\mathrm{Ch}_{i n}^{1}}$ and $\mathrm{Bi}-\mathrm{Ch}_{\text {in }}^{2}$ are two in-plane $\mathrm{Bi}^{-} \mathrm{Ch}_{\text {in }}$ distances, while $\mathrm{Bi}^{-\mathrm{Ch}_{i n}^{i}}$ is the distance between the $\mathrm{BiCh}_{2}$-layers.

\begin{tabular}{|c|c|c|c|c|}
\hline & \multicolumn{2}{|c|}{$\mathrm{LaOBiS}_{2}$} & \multicolumn{2}{|c|}{ LaOBiSSe } \\
\hline & $\mathbf{R}(\AA)$ & $\sigma^{2}\left(\AA^{2}\right)$ & $\mathbf{R}(\AA)$ & $\sigma^{2}\left(\AA^{2}\right)$ \\
\hline $\mathrm{Bi}-\mathrm{S}_{\text {out }}$ & 2.47 & 0.003 & 2.48 & 0.004 \\
\hline $\mathrm{Bi}-\mathrm{S} / \mathrm{Se}_{i n}^{1}$ & 2.68 & 0.003 & 2.82 & 0.003 \\
\hline $\mathrm{Bi}-\mathrm{S} / \mathrm{Se}_{i n}^{2}$ & 3.09 & 0.005 & 3.04 & 0.006 \\
\hline $\mathrm{Bi}-\mathrm{S} / \mathrm{Se}_{i n}^{i}$ & 3.42 & 0.006 & 3.47 & 0.009 \\
\hline
\end{tabular}


metal atoms choose to take the off-center position, forming $s-p$ hybridization to obtain energy gain in the electronic system. We think this idea can be also applicable in the current $\mathrm{BiCh}_{2}$ system, as is the case of $\mathrm{Bi}_{2} \mathrm{O}_{3}, \mathrm{Bi}_{2} \mathrm{~S}_{3}$, and $\mathrm{Bi}_{2} \mathrm{Se}_{3}{ }^{38}$ Indeed, the overall energy position of calculated Se $4 p$ pDOS is higher compared with that of S $3 p$ pDOS in Figs. 3(a) and 3(b). Therefore, although detailed bond analysis ${ }^{38}$ on this series of the material should be important to conclude, the manipulation of the lone pair activity by chalcogen substitution could reduce the local distortion in the conducting layer and recover the conductivity. In the parent compound $\mathrm{LaOBiS}_{2}$, the contribution of electrons to thermal conductivity $\left(\kappa_{e}\right)$ is small compared with that of phonons $\left(\kappa_{p h}\right){ }^{12}$ The drastic decrease of resistivity increases $\kappa_{e}$ but it seems to be canceled by the decrease of $\kappa_{p h}$ with help of possible rattling motion of $\mathrm{Bi}^{39}$ As a result, the $Z T$ value would have benefitted from chalcogen substitution. Such a control of the local structural distortion in the compounds with the lone pair (such as $\mathrm{Bi}, \mathrm{Pb}, \mathrm{Sn}$ ) can be beneficial for further improvement of thermoelectric property.

\section{CONCLUSION}

In summary, we have studied the Se-substitution effect on the electronic and the local structures of the $\mathrm{LaOBiCh}_{2}$ system. It has turned out that the top of the valence band is significantly influenced by the substitution, where pDOS of the in-plane chalcogen atom is dominant. We have also observed that the local structural distortion in the $\mathrm{Bi}$-chalcogen plane is suppressed by Se substitution, which is in accordance with the lone pair activity of the system. It is suggested that controlling the local structural distortion can help enhancing the functionality of thermoelectric materials.

\section{ACKNOWLEDGMENTS}

We thank ESRF staff for support in the EXAFS data collection. The authors would like to thank S. Onari for the use of the workstation. K.T. and T.W. would like to acknowledge the hospitality at the Sapienza University of Rome. This research was partially supported by the Program for Promoting the Enhancement of Research University from MEXT, the Program for Advancing Strategic International Networks to Accelerate the Circulation of Talented Researchers from JSPS (R2705), and JSPS KAKENHI (Nos. 15H03691, 16H04493). This work is a part of the executive protocol of the general agreement for cooperation between the Sapienza University of Rome and Okayama University, Japan.

\section{REFERENCES}

1Y. Mizuguchi, S. Demura, K. Deguchi, Y. Takano, H. Fujihisa, Y. Gotoh, H. Izawa, and O. Miura, J. Phys. Soc. Jpn. 81, 114725 (2012).

2J. Xing, S. Li, X. Ding, H. Yang, and H. H. Wen, Phys. Rev. B 86, 214518 (2012).

${ }^{3}$ R. Jha, A. Kumar, S. K. Singh, and V. P. S. Awana, J. Sup. Novel Mag. 26, 499 (2013).

${ }^{4}$ S. Demura, Y. Mizuguchi, K. Deguchi, H. Okazaki, H. Hara, T. Watanabe, S. J. Denholme, M. Fujioka, T. Ozaki, H. Fujihisa, Y. Gotoh, O. Miura, T. Yamaguchi, H. Takeya, and Y. Takano, J. Phys. Soc. Jpn. 82, 033708 (2013).
${ }^{5_{H}}$ H.-F. Zhai, Z.-T. Tang, H. jiang, K. Xu, K. Zhang, P Zhang, J.-K. Bao, Y.-L. Sun, W.-H. Jiao, I. Nowik, I. Felner, Y.-K. Li, X.-F. Xu, Q. Tao, C.-M. Feng, Z.-A. Xu, and G.-H. Cao, Phys. Rev. B 90, 064518 (2014).

${ }^{6}$ R. Jha, B. Tiwari, and V. P. S. Awana, J. Appl. Phys. 117, 013901 (2015).

${ }^{7}$ D. Yazici, K. Huang, B. D. White, I. Jeon, V. W. Burnett, A. J. Friedman, I. K. Lum, M. Nallaiyan, S. Spagna, and M. B. Maple, Phys. Rev. B 87, 174512 (2013).

${ }^{8}$ R. Sogabe, Y. Goto, and Y. Mizuguchi, Appl. Phys. Express 11, 053102 (2018).

${ }^{9}$ Y. Hijikata, T. Abe, C. Moriyoshi, Y. Kuroiwa, Y. Goto, A. Miura, K. Tadanaga, Y. Wang, O. Miura, and Y. Mizuguchi, J. Phys. Soc. Jpn. 86, 124802 (2017).

${ }^{10}$ Y. Li, X. Lin, L. Li, N. Zhou, X. Xu, C. Cao, J. Dai, L. Zhang, Y. Luo, W. Jiao, Q. Tao, G. Cao, and Z. Xu, Supercond. Sci. Technol. 27, 035009 (2014).

${ }^{11}$ A. Krzton-Mapioza, Z. Guguchia, E. Pomjakushina, V. Pomjakushin, R. Khasanov, H. Luetkens, P. Biswas, A. Amato, H. Keller, and K. Conder, J. Phys. Condens. Matter 26, 215702 (2014).

${ }^{12}$ Y. Mizuguchi, A. Omachi, Y. Goto, Y. Kamihara, M. Matoba, T. Hiroi, J. Kajitani, and O. Miura, J. Appl. Phys. 116, 163915 (2014).

${ }^{13}$ Y. Mizuguchi, A. Nishida, A. Omachi, and O. Miura, Cogent Phys. 3, 1156281 (2016).

${ }^{14}$ A. Nishida, H. Nishiate, C.-H. Lee, O. Miura, and Y. Mizuguchi, J. Phys. Soc. Jpn. 85, 074702 (2016).

${ }^{15}$ T. Sugimoto, E. Paris, T. Wakita, K. Terashima, T. Yokoya, A. Barinov, J. Kajitani, R. Higashinaka, T. D. Matsuda, Y. Aoki, T. Mizokawa, and N. L. Saini, Sci. Rep. 8, 2011 (2018).

${ }^{16}$ Q. Liu, X. Zhang, and A. Zunger, Phys. Rev. B 93, 174119 (2016).

${ }^{17}$ X. Zhou, Q. Liu, J. A. Waugh, H. Li, T. Nummy, X. Zhang, X. Zhu, G. Cao, A. Zunger, and D. S. Dessau, Phys. Rev. B 95, 075118 (2017).

${ }^{18}$ Y. Mizuguchi, A. Miura, A. Nishida, O. Miura, K. Tadanaga, N. Kumada, C. H. Lee, E. Magome, C. Moriyoshi, and Y. Kuroiwa, J. Appl. Phys. 119, 155103 (2016).

${ }^{19}$ P. Blaha, K. Schwarz, G. K. H. Madsen, D. Kvasnicka, and J. Luitz, WIEN2k: An Augmented Plane Wave Plus Local Orbitals Program for Calculating Crystal Properties, Technische Universität Wien, Vienna, 2001.

${ }^{20}$ A. D. Becke and E. R. Johnson, J. Chem. Phys. 124, 221101 (2006).

${ }^{21}$ F. Tran and P. Blaha, Phys. Rev. Lett. 102, 226401 (2009).

${ }^{22}$ M. Ochi, H. Usui, and K. Kuroki, Phys. Rev. Appl. 8, 064020 (2017).

${ }^{23}$ The electronic structure under the monoclinic crystal structure has been calculated by Ref. 22, where splitting of bands in the order of $0.1 \mathrm{eV}$ has been predicted.

${ }^{24}$ A. Athauda, J. Yang, S. Lee, Y. Mizuguchi, K. Deguchi, Y. Takano, O. Miura, and D. Louca, Phys. Rev. B 91, 144112 (2015).

${ }^{25}$ R. Sagayama, H. Sagayama, R. Kumai, Y. Murakami, T. Asano, J. Kajitani, R. Higashinaka, T. D. Matsuda, and Y. Aoki, J. Phys. Soc. Jpn. 84, 123703 (2015).

${ }^{26}$ K. Nagasaka, A. Nishida, R. Jha, J. Kajitani, O. Miura, R. Higashinaka, T. D. Matsuda, Y. Aoki, A. Miura, C. Moriyoshi, Y. Kuroiwa, H. Usui, K. Kuroki, and Y. Mizuguchi, J. Phys. Soc. Jpn. 86, 074701 (2017).

${ }^{27} \mathrm{G}$. Bunker, Introduction to XAFS (Cambridge University Press, 2010).

${ }^{28}$ S. Nagira, J. Sonoyama, T. Wakita, M. Sunagawa, Y. Izumi, T. Muro, H. Kumigashira, M. Oshima, K. Deguchi, H. Okazaki, Y. Takano, O. Miura, Y. Mizuguchi, K. Suzuki, H. Usui, K. Kuroki, K. Okada, Y. Muraoka, and T. Yokoya, J. Phys. Soc. Jpn. 83, 033703 (2014).

${ }^{29}$ A. Miura, Y. Mizuguchi, T. Takei, N. Kumada, E. Magome, C. Moriyoshi, Y. Kuroiwa, and K. Tadanaga, Solid State Commun. 227, 19 (2016).

${ }^{30}$ G. Wang, D. Wang, X. Shi, and Y. Peng, Mod. Phys. Lett. B 31, 1750265 (2017).

${ }^{31}$ S. M. Goldberg, C. S. Fadley, and S. Kono, J. Electron. Spectrosc. Relat. Phenom. 21, 285 (1981).

${ }^{32}$ J. J. Yeh and I. Lindau, Atom. Data Nucl. Data 32, 1 (1985). 
${ }^{33}$ E. Paris, Y Mizuguchi, M. Y. Hacisalihoglu, T Hiroi, B Joseph, G Aquilanti, O Miura, T Mizokawa, and N. L. Saini, J. Phys. Condens. Matter 29, 145603 (2017). ${ }^{34}$ E. Paris, Y. Mizuguchi, T. Wakita, K. Terashima, T. Yokoya, T. Mizokawa, and N. L. Saini, J. Phys. Condens. Matter 30, 455703 (2018).

${ }^{35}$ A. L. Ankudinov, B. Ravel, J. J. Rehr, and S. D. Conradson, Phys. Rev. B 58, 7565 (1998); J. J. Rehr and R. C. Albers, Rev. Mod. Phys. 72, 621 (2000).

${ }^{36}$ T. Ressler, J. Synch. Rad. 5, 118 (1998).
${ }^{37}$ A. Walsh, D. J. Payne, R. G. Egdell, and G. W. Watson, Chem. Soc. Rev. 40, 4455 (2011).

${ }^{38}$ A. Walsh, G. W. Watson, D. J. Payne, R. G. Edgell, J. Guo, P.-A. Glans, T. Learmonth, and K. E. Smith, Phys. Rev. B 73, 235104 (2006).

${ }^{39}$ C. H. Lee, A. Nishida, T. Hasegawa, H. Nishiate, H. Kunioka, S. Ohira-Kawamura, M. Nakamura, K. Nakajima, and Y. Mizuguchi, Appl. Phys. Lett. 112, 023903 (2018). 